\title{
MEAN FLOWS GENERATED BY A PROGRESSING WATER WAVE PACKET
}

\author{
R. GRIMSHAW \\ (Received 25 February 1980) \\ (Revised 8 May 1980)
}

\begin{abstract}
Equations are derived which describe the evolution of the mean flow generated by a progressing water wave packet. The effect of friction is included, and so the equations are subject to the boundary conditions first derived by Longuet-Higgins [10]. Solutions of the equations are obtained for a wave packet of finite length, and also for a uniform wave train. The latter solution is compared with experiments.
\end{abstract}

\section{Introduction}

It was shown by Stokes [15] that for an inviscid progressive water wave of small amplitude $a$, the fluid particles do not exactly describe closed paths; in addition to their orbital motion there is an $O\left(a^{2}\right)$ drift velocity in the direction of the wave propagation. For water of mean depth $h$ this Stokes drift velocity is given by

$$
\bar{u}^{S}=\frac{a^{2} 2 \omega \kappa \cosh 2 \kappa(z+h)}{\sinh ^{2} \kappa h}+O\left(a^{4}\right)
$$

where $\omega$ is the wave frequency, $\kappa$ is the wavenumber, $4 a$ is the crest-to-trough amplitude and $z$ is the vertical co-ordinate (measured upwards from the mean free surface). If $\bar{u}$ is the mean Eulerian velocity (that is, the mean recorded at a fixed place), then the mean Lagrangian, or mass transport, velocity is $\bar{u}^{L}=\bar{u}+$ $\bar{u}^{s}$. In the interior of the fluid, the $O\left(a^{2}\right)$ equation governing the mean Eulerian 
velocity is

$$
\frac{\partial \bar{u}}{\partial t}+\frac{\partial \bar{q}}{\partial x}=\nu \frac{\partial^{2} \bar{u}}{\partial z^{2}}
$$

where $x$ is a horizontal co-ordinate in the direction of the waves, $t$ is the time, $\bar{q}$ is a mean pressure (independent of $z$ ), and $\nu$ is the kinematic viscosity.

Just over one hundred years after Stokes derived the expression (1.1) for the drift velocity, Longuet-Higgins [10] showed that viscous effects in the boundary layers of thickness $(\nu / \omega)^{1 / 2}$ at the bottom and free surface profoundly modify the mean velocity fields. He showed that just outside the bottom boundary layer, there is a mean Eulerian velocity

$$
\lim _{z \rightarrow-h} \bar{u}=\frac{a^{2} 3 \omega \kappa}{\sinh ^{2} \kappa h}
$$

while just outside the free surface boundary layer there is a mean Eulerian velocity gradient

$$
\lim _{z \rightarrow 0} \frac{\partial \bar{u}}{\partial z}=\frac{a^{2} 8 \omega \kappa^{2}}{\tanh \kappa h} .
$$

These expressions arise due to the action of Reynolds stresses transferring momentum across the boundary layer, and although dependent on a non-zero value of $\nu$, are independent of the value of $\nu$. Equations (1.3) and (1.4) act as boundary conditions for the interior mean flow equation (1.2). Experiments by Russell and Onsorio [14] have verified the validity of the bottom boundary condition (1.3), while experiments by Longuet-Higgins [11] have verified the free-surface condition (1.4). When $\bar{u}$ is independent of the time $t$, LonguetHiggins [10] derived the conduction solution to (1.2), (1.3) and (1.4); this solution with $\partial \bar{q} / \partial x$ determined by the constraint of zero mass transport, gives qualitative agreement with the experimental results of Russell and Onsorio [14], although there are some discrepancies (see Figures 1 and 2 in Section 5). Subsequently Chang [4] and Ünlüata and Mei [16] re-derived Longuet-Higgins' results using Lagrangian co-ordinates a priori. All of these authors assumed a uniform wave train so that the amplitude $a$ is a constant. Liu and Davis [9] allowed the amplitude to decay exponentially in time, but their solution contains some anomalies at certain values of the depth $h$. Huang [8] attempted to obtain better agreement with experiment by imposing different stress conditions at the free surface, and Madsen [12] has considered the effect of an imposed wind stress, and the inclusion of the Coriolis terms in (1.2). Longuet-Higgins [10] and Dore [6] have discussed the consequences of including the nonlinear advective terms in the mean flow equation (1.2).

In this paper we propose to consider the mean flow generated by a wave packet. Thus the amplitude of the waves is allowed to depend on both $x$ and $t$, 
and the wave amplitude envelope propagates at the group velocity, being simultaneously attenuated by friction. This has the advantage that we are then able to consider the initial value problem in which the wave packet is initiated at $t=0, x=0$ say, and discuss the subsequent evolution of the mean flow when it is governed by the equations (1.2), (1.3) and (1.4) and an equation for conservation of mass. In Section 2 we formulate the equations of motion in the generalized Lagrangian-mean formulation of Andrews and McIntyre [1]. This has some conceptual advantages, and considerably simplifies the analytical treatment of the free surface, although at the expense of some complications in the viscous terms in the interior. Then in Section 3 we considered a modulated wave packet and derive the wave action equation to describe changes in the wave amplitude. In Section 4 we derive the equations for the evolution of the interior mean flow, which consist of equations (1.2), (1.3), (1.4) and an equation for the conservation of mass. Then in Section 5 we discuss solutions of the mean flow equations. We concentrate on two cases, (i) a uniform wave train and (ii) a wave packet, both initiated by a wavemaker at $x=0$ at $t=0$. We show that in case (ii) for a wave packet the interior solution for the Eulerian mean flow is a depth independent irrotational flow, modified by boundary layers of thickness $(\nu t)^{-1 / 2}$. For water of finite depth, the frictional decay factor for the wave amplitude is proportional to $\nu^{1 / 2}$. It is thus appropriate to consider a wave packet only for times $t$ of $O\left(\nu^{-1 / 2}\right)$, and the boundary layers remain confined for these times. By contrast, for case (i) of a uniform wave train, the wave amplitude is maintained by the wavemaker, and the boundary layers can penetrate to the interior. Ultimately, for times $t$ of $O\left(h^{2} / \nu\right)$, the Longuet-Higgins conduction solution is reached. However, for the experiments of Russell and Onsorio this diffusive time scale of $O\left(h^{2} / v\right)$ is too long, and instead we compare their experimental results with the solution of the interior mean flow equations for times $t$ of $O\left(\nu^{-1 / 2}\right)$. Our results give reasonably good agreement with the experiments.

\section{Generalized Lagrangian-mean formulation}

The Eulerian equations of motion for an incompressible homogeneous fluid are

$$
\frac{\partial u_{i}}{\partial x_{i}}=0
$$

and

$$
\frac{d u_{i}}{d t}+\frac{\partial p}{\partial x_{i}^{\prime}}=\nu \frac{\partial^{2} u_{i}}{\partial x_{j}^{\prime} \partial x_{j}^{\prime}},
$$


where

$$
\frac{d}{d t}=\frac{\partial}{\partial t}+u_{i} \frac{\partial}{\partial x_{i}^{\prime}}
$$

Here $u_{1}$ are the velocity components, $p$ is the reduced pressure, $t$ is the time and $x_{i}^{\prime}$ are Eulerian Cartesian co-ordinates; the Latin index $i$ takes the values 1, 2 or 3 and the summation convention is used; $\delta_{i}$ is the Kroneker "delta and the $x_{3}^{\prime}$-axis is the vertical axis. The variables are non-dimensional, based on a length scale $L$ (a typical wavelength), a time scale $\omega_{1}^{-1}\left(\omega_{1}\right.$ is a typical frequency) and $\omega_{1} L$ is the velocity scale; then $\nu$ is a non-dimensional kinematic viscosity, and the dimensional kinematic viscosity is $\omega_{1} L^{2} \nu$. It is convenient in the subsequent analysis to distinguish between horizontal co-ordinates $x_{\alpha}^{\prime}(\alpha=1,2)$ and the vertical co-ordinates $z=x_{3}^{\prime}$ by employing Greek indices for horizontal variables, while retaining Latin indices for all three co-ordinates; similarly $u_{\alpha}$ are the horizontal velocity components and $w=u_{3}$ is the vertical velocity. It will be assumed that the fluid occupies a horizontal channel, bounded below by a rigid boundary $z^{\prime}=-h\left(x_{\alpha}^{\prime}\right)$ and above by the free surface $z^{\prime}=\zeta\left(x_{\alpha}^{\prime}, t\right)$. The boundary conditions are

$$
\begin{array}{rlrl}
c u_{i} & =0 & \text { on } & z^{\prime}=-h\left(x_{\alpha}^{\prime}\right), \\
\frac{\partial \zeta}{\partial t}+u_{\alpha} \frac{\partial \zeta}{\partial x_{\alpha}^{\prime}}=w & \text { on } & z^{\prime}=\zeta\left(x_{\alpha}^{\prime}, t\right), \\
-p+g \zeta+\frac{2 v n_{i} n_{j}}{n_{k} n_{k}} \frac{\partial u_{t}}{\partial x_{j}^{\prime}}=0 & \text { on } & z^{\prime}=\zeta\left(x_{\alpha}^{\prime}, t\right), \\
\tau_{i}^{(\gamma)}\left(\frac{\partial u_{i}}{\partial x_{j}^{\prime}}+\frac{\partial u_{j}}{\partial x_{i}^{\prime}}\right) n_{j}=0 & \text { on } & z^{\prime}=\zeta\left(x_{\alpha}^{\prime}, t\right), & \gamma=1,2 .
\end{array}
$$

Here $g$ is a non-dimensional gravity (dimensional gravity is $\omega_{1}^{2} L g$ ), $n_{i}$ is a vector normal to the free surface, and $\tau_{l}^{(\gamma)}$ are vectors tangent to the free surface. We put

$$
n_{\alpha}=-\frac{\partial \zeta}{\partial x_{\alpha}^{\prime}}, \quad n_{3}=1
$$

and

$$
\tau_{\alpha}^{(\gamma)}=\delta_{\alpha \gamma}, \quad \tau_{\}}^{(\gamma)}=\frac{\partial \zeta}{\partial x_{\alpha}^{\prime}}
$$

Thus (2.2c) is the condition that the normal stress vanish at the free surface, while (2.2d) is the condition that the tangential stress vanish.

To describe a modulated progressing wave packet we introduce a small parameter $\varepsilon$, and define

$$
X_{\alpha}^{\prime}=\varepsilon x_{\alpha}^{\prime} \quad \text { and } \quad T^{\prime}=\varepsilon t
$$


Then, if $\phi$ is any field variable, we put

$$
\phi\left(x_{i}^{\prime}, t\right)=\bar{\phi}\left(X_{\alpha}^{\prime}, T ; z^{\prime}\right)+\phi^{\prime}\left(X_{\alpha}^{\prime}, T ; z^{\prime}, \theta^{\prime}\right),
$$

and

$$
\theta^{\prime}=\frac{1}{\varepsilon} \Theta\left(X_{\alpha}^{\prime}, T\right)
$$

where $\phi^{\prime}$ is periodic in the phase $\theta^{\prime}$ with period $2 \pi$ and zero mean. Eulerian mean, $\bar{\phi}$, is an $O\left(a^{2}\right)$ quantity which varies in the horizontal, and in time, on the scale $\varepsilon^{-1}$, while $\phi^{\prime}$, the Eulerian perturbation, is an $O(a)$ wave-like quantity; here $a$ is a small parameter which measures the wave amplitude. Consistently with these hypotheses, we assume that $\nu$ is also a small parameter and scales with either $\varepsilon$ (in the deep water approximation), or $\varepsilon^{2}$ (otherwise), while the depth $h$ is $h\left(X_{\alpha}^{\prime}\right)$. Next we note that

$$
\bar{\phi}\left(X_{i}^{\prime}, t\right)=\left\langle\phi\left(X_{i}^{\prime}, t\right)\right\rangle=\frac{1}{2 \pi} \int_{0}^{2 \pi} \phi d \theta^{\prime}
$$

defines an averaging operator, which represents a local average over the phase of the waves. Substitution of expressions like (2.5a) into (2.1) and (2.2) and linearization would then determine the wave-like quantities, and in particular the variation of the wave amplitude on the length and time scales $\varepsilon^{-1}$. Application of the averaging operator to (2.1) and (2.2) gives the equations governing the Eulerian mean flow, whose forcing terms can be evaluated correct to $O\left(a^{2}\right)$. In both cases boundary layers of thickness $\nu^{1 / 2}$ are inserted at $z=-h$ and $z=\zeta$ to ensure that all the boundary conditions are satisfied. This is essentially the procedure used by Longuet-Higgins [10] for a uniform wave train, and by Liu and Davis [19] for an attenuating wave train. The mass transport is found $a$ posteriori by calculating the Stokes corrections and adding these to the Eulerian mean flow.

However, instead of using this Eulerian procedure outlined above, we shall use the generalized Lagrangian-mean flow (GLM) formulation recently proposed by Andrews and McIntyre [1,2]. In the present context, a Lagrangian formulation has some practical and conceptual advantages, although a major disadvantage is the complicated form that the viscous terms take in Lagrangian co-ordinates. Chang [4], for deep water waves, and Ünlüata and Mei [16] used the traditional Lagrangian co-ordinates. Grimshaw [7] used the GLM formulation to discuss mean flows induced by internal gravity waves in a shear flow, and we shall use a very similar formulation here. Let $x_{i}$ be generalized Lagrangian co-ordinates, and let $\xi_{l}(x, t)$ be particle displacements defined so that

$$
x_{i}^{\prime}=x_{i}+\xi_{i}
$$

Occasionally we shall distinguish the vertical particle displacement and put 
$\xi_{3}=\eta$. We then define a Lagrangian-mean operator by

$$
\bar{\phi}^{L}\left(x_{i}, t\right)=\left\langle\phi\left(x_{i}+\xi_{i}, t\right)\right\rangle .
$$

In physical terms, $\bar{\phi}$, the Eulerian mean, is the average over the phase of the waves taken at a fixed place, while $\bar{\phi}^{L}$, the Lagrangian mean, is the average over the phase of the waves following the fluid motion. As shown by Andrews and McIntyre [1], this latter motion is made precise by requiring that

$$
\left\langle\xi_{i}\right\rangle=0 .
$$

Like $\bar{\phi}, \bar{\phi}^{L}$ is an $O\left(a^{2}\right)$ quantity. Thus $x_{i}$ is a co-ordinate which moves with the Lagrangian mean velocity $\bar{u}_{i}^{L}$ whenever the co-ordinate $x_{i}^{\prime}$ moves with the true velocity $u_{i}$. Since $\xi_{i}$ is wave-like and $O(a)$, it follows from $(2.5 \mathrm{a}, \mathrm{b})$ and $(2.7)$ that we may put

$$
\phi\left(x_{i}, t\right)=\bar{\phi}^{L}\left(X_{\alpha}, T ; z\right)+\hat{\phi}\left(X_{\alpha}, T ; z, \theta\right),
$$

where

$$
\theta=\frac{1}{\varepsilon} \Theta\left(X_{a}, T\right),
$$

and

$$
X_{\alpha}=\varepsilon x_{\alpha} .
$$

Here $\hat{\phi}$ is periodic in $\theta$ with period $2 \pi$ and zero mean. Then we may show that

$$
\hat{\phi}=\phi^{\prime}+O\left(a^{2}\right),
$$

and

$$
\bar{\phi}^{L}=\bar{\phi}+\bar{\phi}^{s},
$$

where

$$
\bar{\phi}^{s}=\left\langle\xi_{i} \frac{\partial \phi^{\prime}}{\partial x_{i}}\right\rangle+O\left(a^{4}\right),
$$

and $\bar{\phi} s$ is usually the "Stokes correction". Finally we note the useful property that, in the GLM formulation,

$$
\frac{d \phi}{d t}=\frac{\partial \phi}{\partial t}+\bar{u}_{i}^{L} \frac{\partial \phi}{\partial x_{i}}
$$

and so

$$
\left\langle\frac{d}{d t}\right\rangle=\frac{d}{d t}\langle\phi\rangle
$$

The next step is to obtain the equations of motion in Lagrangian form. First we introduce the Jacobian

$$
J=\operatorname{det}\left\{\frac{\partial x_{i}^{\prime}}{\partial x_{j}}\right\}
$$


Then (2.1a) implies that $J$ is a mean quantity $(\hat{J}=0)$ and

$$
\frac{1}{J} \frac{d J}{d t}+\frac{\partial \bar{u}_{i}^{L}}{\partial x_{i}}=0
$$

Substituting (2.7) into (2.13) and expanding in $\xi$, it follows that

$$
\frac{\partial \xi_{i}}{\partial x_{i}}=O\left(a^{2}\right)
$$

and

$$
\frac{\partial \bar{u}_{i}^{L}}{\partial x_{i}}=\frac{\partial}{\partial t}\left\langle\frac{1}{2} \frac{\partial^{2}}{\partial x_{i} \partial x_{j}}\left(\xi_{i} \xi_{j}\right)\right\rangle+O\left(a^{4}\right) .
$$

Next, the equation of motion becomes, after multiplying by $\partial x_{i}^{\prime} / \partial x_{j}$,

$$
\frac{\partial x_{i}^{\prime}}{\partial x_{j}} \frac{d \bar{u}_{i}^{L}}{d t}+\frac{\partial x_{i}^{\prime}}{\partial x_{j}} \frac{d^{2} \xi_{i}}{d t^{2}}+\frac{\partial p}{\partial x_{j}}=\nu \frac{\partial x_{i}^{\prime}}{\partial x_{j}} \frac{\partial^{2} u_{i}}{\partial x_{k}^{\prime} \partial x_{k}^{\prime}},
$$

where

$$
u_{i}=\bar{u}_{i}^{L}+\hat{u}_{i} \quad \text { and } \quad \hat{u}_{i}=\frac{d \xi_{i}}{d t} .
$$

The right-hand side of (2.16) can be expressed in Lagrangian terms by introducing $K_{i j}$, the $i$-jth co-factor of $J$, so that

$$
K_{i j} \frac{\partial x_{i}^{\prime}}{\partial x_{k}}=K_{j i} \frac{\partial x_{k}^{\prime}}{\partial x_{i}}=\delta_{j k} J
$$

It may then be shown that

$$
K_{i j}=J \frac{\partial x_{j}}{\partial x_{i}^{\prime}} \quad \text { and } \quad \frac{\partial K_{i j}}{\partial x_{j}}=0 .
$$

Hence the viscous force in (2.16) may be put in the form

$$
\nu \frac{\partial x_{i}^{\prime}}{\partial x_{j}} \frac{\partial^{2} u_{i}}{\partial x_{k}^{\prime} \partial x_{k}^{\prime}}=\frac{\nu}{J} \frac{\partial x_{i}^{\prime}}{\partial x_{j}} \frac{\partial}{\partial x_{r}}\left(\frac{K_{k r} K_{k s}}{J} \frac{\partial u_{i}}{\partial x_{s}}\right) .
$$

Extracting the mean and perturbed parts of (2.16) it follows that

$$
\frac{\partial^{2} \xi_{i}}{\partial t^{2}}+\frac{\partial \hat{p}}{\partial x_{i}}=\nu \frac{\partial^{2} \hat{u}_{i}}{\partial x_{k} \partial x_{k}}+O\left(a^{2}\right)
$$

and

$$
\frac{\partial \bar{u}_{i}^{L}}{\partial t}+\frac{\partial \bar{p}^{L}}{\partial x_{i}}=\nu \frac{\partial^{2} \bar{u}_{i}^{L}}{\partial x_{k} \partial x_{k}}+R_{i}^{L}
$$


where

$$
\begin{aligned}
R_{i}{ }^{L}= & \frac{\partial}{\partial t}\left\langle-\hat{u}_{j} \frac{\partial \xi_{j}}{\partial x_{i}}\right\rangle+\frac{\partial}{\partial x_{i}}\left\langle\frac{1}{2} \hat{u}_{j} \hat{u}_{j}\right\rangle+\nu\left\langle\frac{\partial \xi_{j}}{\partial x_{i}} \frac{\partial^{2} \hat{u}_{j}}{\partial x_{k} \partial x_{k}}\right) \\
& +\nu \frac{\partial}{\partial x_{r}}\left(-\left(\frac{\partial \xi_{s}}{\partial x_{r}}+\frac{\partial \xi_{r}}{\partial x_{s}}\right) \frac{\partial u_{i}}{\partial x_{s}}\right)+O\left(a^{4}\right)
\end{aligned}
$$

and

$$
\hat{u}_{i}=\frac{\partial \xi_{i}}{\partial t}+O\left(a^{2}\right)
$$

Thus the equations to be solved for the perturbed, wave-like terms are (2.15a) and (2.20a), while the equations for the Lagrangian mean flow are (2.15b) and (2.20b).

The equations for the Eulerian mean flow may be obtained directly and simply from (2.1), or with more difficulty by calculating the "Stokes corrections" from (2.11c). Thus, for example,

$$
\bar{u}_{i}^{s}=\left\langle\xi_{j} \frac{\partial \hat{u}_{i}}{\partial x_{j}}\right\rangle+O\left(a^{4}\right) .
$$

Then substituting expressions such as (2.11b) for $\bar{u}_{t}^{L}$ and $\bar{p}^{L}$ into (2.15b) and (2.20b) it may be shown that

$$
\frac{\partial \bar{u}_{t}}{\partial x_{t}}=0
$$

and

$$
\frac{\partial \bar{u}_{i}}{\partial t}+\frac{\partial \bar{p}}{\partial x_{i}}=\nu \frac{\partial^{2} \bar{u}_{i}}{\partial x_{k} \partial x_{k}}+R_{i},
$$

where

$$
R_{i}=-\left\langle\hat{u}, \frac{\partial \hat{u}_{i}}{\partial x_{j}}\right\rangle+O\left(a^{4}\right)
$$

Next, the boundary condition $(2.2 \mathrm{a})$ becomes

$$
\vec{u}_{i}^{L}=0 \text { and } \xi_{i}=0 \text { on } z=-h\left(x_{\alpha}\right) .
$$

The free surface in the GLM formulation is described by $z=\bar{\zeta}^{L}(x, t)$ with the Lagrangian perturbation being identically zero. Then the boundary condition (2.2b) becomes

$$
\frac{\partial \bar{\zeta}^{L}}{\partial t}=\bar{w}^{L}+O\left(a^{4}\right) \quad \text { on } z=0,
$$

where we have assumed that the level $z=0$ is chosen so that $\bar{\zeta}^{L}$ is $O\left(a^{2}\right)$. The 
viscous boundary conditions $(2.2 \mathrm{c}, \mathrm{d})$ then become

$$
-\hat{p}+g \eta+2 \nu \frac{\partial \hat{w}}{\partial z}=O\left(a^{2}\right) \quad \text { on } z=0,
$$

and

$$
\frac{\partial \eta}{\partial x_{\alpha}}+\frac{\partial \xi_{\alpha}}{\partial z}=O\left(a^{2}\right) \text { on } z=0,
$$

for the perturbation terms, while the mean flow boundary conditions are

$$
-\bar{p}^{L}+g \bar{\zeta}^{L}+2 \nu \frac{\partial \bar{w}^{L}}{\partial z}=2 \nu\left\langle\frac{\partial \hat{w}}{\partial x_{i}} \frac{\partial \xi_{i}}{\partial z}\right\rangle+O\left(a^{4}\right), \quad \text { on } z=0
$$

and

$$
\frac{\partial \bar{w}^{L}}{\partial x_{\alpha}}+\frac{\partial \bar{u}_{\alpha}^{L}}{\partial z}=\frac{\partial}{\partial t}\left\langle\frac{\partial \eta}{\partial x_{\beta}} \frac{\partial \xi_{\beta}}{\partial x_{\alpha}}+\frac{\partial \eta}{\partial x_{\alpha}} \frac{\partial \xi_{\beta}}{\partial x_{\beta}}\right\rangle+O\left(a^{4}\right), \quad \text { on } z=0
$$

where we have used $(2.15 a)$ to simplify $(2.26 a, b)$. In Eulerian terms, the free surface is prescribed by $z^{\prime}=\zeta$, where $\zeta$ is decomposed into $\bar{\zeta}+\zeta^{\prime}$ of (2.5a). Then it is readily shown that

$$
\eta=\zeta^{\prime}+O\left(a^{2}\right) \text { on } z=0,
$$

and

$$
\bar{\zeta}^{L}=\bar{\zeta}+\left\langle\xi_{\alpha} \frac{\partial \zeta^{\prime}}{\partial x_{\alpha}}\right\rangle+O\left(a^{4}\right) \text { on } z=0 .
$$

In the Eulerian formulation, the boundary conditions for the mean flow are

$$
\begin{aligned}
\bar{u}_{i}= & 0 \text { on } z=-h\left(x_{\alpha}\right), \\
\frac{\partial \bar{\zeta}}{\partial t}-\bar{w}= & -\frac{\partial}{\partial x_{\alpha}}\left\langle\eta \hat{u}_{\alpha}\right\rangle+O\left(a^{4}\right) \quad \text { on } z=0, \\
-\bar{p}+g \bar{\zeta}+2 \nu \frac{\partial \bar{w}}{\partial z}= & -\left\langle\xi_{i} \frac{\partial \hat{u}_{i}}{\partial t}\right\rangle-g\left\langle\xi_{\alpha} \frac{\partial \eta}{\partial x_{\alpha}}\right\rangle \\
& +\nu\left\langle\xi_{j}\left(\frac{\partial^{2} \hat{u}_{j}}{\partial x_{k} \partial x_{k}}-2 \frac{\partial^{2} \hat{w}}{\partial x_{j} \partial z}\right)\right\rangle+O\left(a^{4}\right) \text { on } z=0,
\end{aligned}
$$

and

$$
\begin{aligned}
\frac{\partial \bar{w}}{\partial x_{\alpha}}+\frac{\partial \bar{u}_{\alpha}}{\partial z}= & \left\langle 2 \frac{\partial \eta}{\partial x_{\alpha}} \frac{\partial u_{\beta}}{\partial x_{\beta}}-\eta \frac{\partial^{2} \hat{u}_{\alpha}}{\partial x_{k} \partial x_{k}}\right\rangle+\frac{\partial}{\partial x_{\beta}}\left\langle\eta\left(\frac{\partial u_{\beta}}{\partial x_{\alpha}}+\frac{\partial u_{\alpha}}{\partial x_{\beta}}\right)\right\rangle \\
& +O\left(a^{4}\right) \text { on } z=0 .
\end{aligned}
$$

In summary, the equations to be solved for the perturbation variables are (2.15a) and (2.20a), with the boundary conditions (2.23) and (2.25a, b). The 
Lagrangian mean flow equations are (2.15b) and (2.20b), with the boundary conditions (2.23), (2.24) and (2.26a, b), while the alternative Eulerian mean flow equations are $(2.22 \mathrm{a}, \mathrm{b})$, with the boundary conditions $(2.28 \mathrm{a}, \mathrm{b}, \mathrm{c}, \mathrm{d})$. To this point we have used the smallness of the amplitude parameter $a$ but have made no explicit use of the smallness of $\varepsilon$, and the equations are exact to all orders in $\varepsilon$.

\section{Modulated waves}

In this section we shall seek an asymptotic solution to the perturbation equations (2.15a) and (2.20a), and the boundary conditions (2.23) and (2.25a, b), of the following form:

$$
\begin{aligned}
& \xi_{j}=a\left\{\xi_{j}^{(0)}\left(X_{\alpha}, T ; z\right)+\varepsilon \xi_{j}^{(1)}\left(X_{\alpha}, T ; z\right)+O\left(\varepsilon^{2}\right)\right\} \exp (i \theta)+\text { c.c., } \\
& \hat{p}=a\left\{\hat{p}^{(0)}\left(X_{\alpha}, T ; z\right)+\varepsilon \hat{p}^{(1)}\left(X_{\alpha}, T ; z\right)+O\left(\varepsilon^{2}\right)\right\} \exp (i \theta)+\text { c.c.. }
\end{aligned}
$$

Here c.c. denotes complex conjugate and $\theta$ is defined by (2.10b). Equations $(3.1 \mathrm{a}, \mathrm{b})$ describe a modulated progressing wave packet whose local frequency $\omega$ and local wave number are defined by

$$
\omega=-\frac{\partial \Theta}{\partial T} \quad \text { and } \quad \kappa_{\alpha}=\frac{\partial \Theta}{\partial X_{\alpha}} .
$$

Also we define $\kappa$ by $\kappa^{2}=\kappa_{\alpha} \kappa_{\alpha}$. Substituting $(3.1 \mathrm{a}, \mathrm{b})$ into $(2.15 \mathrm{a})$ and $(2.20 \mathrm{~b})$ it follows that

$$
\begin{aligned}
\xi_{\alpha}^{(0)} & =\frac{i \kappa_{\alpha} \cosh \kappa(z+h)}{\kappa \sinh \kappa h} A\left(X_{\alpha}, T\right), \\
\eta^{(0)} & =\frac{\sinh \kappa(z+h)}{\sinh \kappa h} A\left(X_{\alpha}, T\right),
\end{aligned}
$$

and

$$
\hat{p}^{(0)}=\frac{\omega^{2} \cosh \kappa(z+h)}{\kappa \sinh \kappa h} A\left(X_{\alpha}, T\right) .
$$

Here $A\left(X_{\alpha}, T\right)$ is the amplitude of the free surface displacement. We have also satisfied the boundary condition $\eta^{(0)}=0$ at $z=-h$, as, to $O(\varepsilon)$, this is the kinematic and non-viscous part of the boundary condition (2.23). Recalling that $\nu$ scales with either $\varepsilon$ (deep water approximation) or $\varepsilon^{2}$ (otherwise), the boundary condition (2.25a) will be satisfied if and only if

$$
\omega^{2}=g \kappa \tanh \kappa h,
$$

which is the well known dispersion relation for water waves. Recalling (3.2), equation (3.4) is here a partial differential equation for the phase $\Theta$. At the next 
order in $\varepsilon$ we obtain the equations

$$
\begin{aligned}
i \kappa_{\alpha} \xi_{\alpha}^{(1)}+\frac{\partial \eta^{(1)}}{\partial z} & =I, \\
-\omega^{2} \xi^{(1)}+i \kappa_{\alpha} \hat{p}^{(1)} & =F_{\alpha},
\end{aligned}
$$

and

$$
-\omega^{2} \eta^{(1)}+\frac{\partial \hat{p}^{(1)}}{\partial \eta}=F_{3} .
$$

Here the right-hand sides of $(3.5 \mathrm{a}, \mathrm{b}, \mathrm{c})$ are known in terms of $\xi_{i}^{(0)}$ and $\hat{p}^{(0)}$, and are given by

$$
\begin{aligned}
I & =-\frac{\partial \xi_{\alpha}^{(0)}}{\partial X_{\alpha}} \\
F_{\alpha} & =2 i \omega \frac{\partial \xi_{\alpha}^{(0)}}{\partial T}+i \frac{\partial \omega}{\partial T} \xi_{\alpha}^{(0)}-\frac{\partial \hat{p}^{(0)}}{\partial X_{\alpha}}
\end{aligned}
$$

and

$$
F_{3}=2 i \omega \frac{\partial \eta^{(0)}}{\partial T}+i \frac{\partial \omega}{\partial T} \eta^{(0)}
$$

There is no contribution from the viscous term in (2.20a) as the zero-order solution $(3.3 \mathrm{a}, \mathrm{b})$ is irrotational. Further, it may be shown from $(3.5 \mathrm{~b}, \mathrm{c})$ and $(3.6 \mathrm{~b}, \mathrm{c})$ that the first order solution is also irrotational. Eliminating $\xi_{\alpha}^{(1)}$ and $\hat{p}^{(1)}$ it follows that

$$
\omega^{2}\left(\frac{\partial^{2} \eta^{(1)}}{\partial z^{2}}-\kappa^{2} \eta^{(1)}\right)=M
$$

where

$$
M=\kappa^{2} F_{3}+\frac{\partial}{\partial z}\left(\omega^{2} I+i \kappa_{\alpha} F_{\alpha}\right) .
$$

The full viscous boundary conditions (2.23) and (2.25a, b) cannot be satisfied by the solution (3.3), which is an inner solution, and must be supplemented by boundary layer solutions at $z=0$ and $z=-h$. Consider the bottom boundary layer first. The boundary layer thickness is $\nu^{1 / 2}$, and so we introduce the boundary layer variables

$$
z^{*}=\frac{(z+h)}{\nu^{1 / 2}}, \quad \eta^{*}=\frac{z}{\nu^{1 / 2}}, \quad \xi_{\alpha}^{*}=\xi_{\alpha} \quad \text { and } \quad \hat{p}^{*}=\hat{p} .
$$

Substituting these variables into (2.15a) and (2.20a), the leading terms in the boundary layer equations are

$$
\frac{\partial \xi^{*}}{\partial x_{\alpha}}+\frac{\varepsilon}{\nu^{1 / 2}} \frac{\partial h}{\partial X_{\alpha}} \frac{\partial \xi_{\alpha}^{*}}{\partial z^{*}}+\frac{\partial \eta^{*}}{\partial z^{*}}=0
$$




$$
\frac{\partial^{2} \xi_{\alpha}^{*}}{\partial t^{2}}+\frac{\partial \hat{p}^{*}}{\partial x_{\alpha}}+\frac{\varepsilon}{\nu^{1 / 2}} \frac{\partial h}{\partial X_{\alpha}} \frac{\partial \hat{p}^{*}}{\partial z^{*}}=\frac{\partial^{2}}{\partial z^{* 2}} \frac{\partial \xi_{\alpha}^{*}}{\partial t}
$$

and

$$
\frac{\partial p^{*}}{\partial z^{*}}=0
$$

The boundary conditions are (2.23), or

$$
\xi_{\alpha}^{*}=0 \text { and } \eta^{*}=0 \text { on } z^{*}=0,
$$

and the matching conditions with the interior solutions are

$$
\lim _{z^{*} \rightarrow \infty}\left\{\xi_{\alpha}^{*}, \eta^{*}, \hat{p}^{*}\right\}=\lim _{z \rightarrow-h}\left\{\xi_{\alpha}, \nu^{-1 / 2} \eta, \hat{p}\right\} \text {. }
$$

Equation (3.9c) and the matching condition (3.11) show that $\hat{p}^{*}=\hat{p}$ within the boundary layer where $\hat{p}$ is evaluated at $z=-h$. Next, we seek a solution of (3.9b) for $\xi_{\alpha}^{*}$ which is proportional to $\exp (i \theta)$, and satisfies the boundary condition (3.10) and the matching condition (3.4). The result is

$$
\xi_{\alpha}^{*}=a \xi_{\alpha}^{(0)} \exp (i \theta)\left\{1-\exp \left(-\gamma z^{*}\right)\right\}+\text { c.c., }
$$

where

$$
\gamma=|\omega|^{1 / 2} \exp \left(-\frac{i \pi}{4} \operatorname{sign} \omega\right) \text {. }
$$

Finally, $\eta^{*}$ is found from (3.9a) and (3.10) which give

$$
\begin{aligned}
\eta^{*}= & -a i \kappa_{\alpha} \xi_{\alpha}^{(0)} \exp (i \theta)\left\{z^{*}+\gamma^{-1}\left[\exp \left(-\gamma z^{*}\right)-1\right]\right\} \\
& -\frac{a \varepsilon}{\nu^{1 / 2}} \frac{\partial h}{\partial X_{\alpha}} \xi_{\alpha}^{(0)} \exp (i \theta)\left\{1-\exp \left(-\gamma z^{*}\right)\right\}+\text { c.c. }
\end{aligned}
$$

In both (3.12a) and (3.13) $\xi_{\alpha}^{(0)}$ is evaluated at $z=-h$. The matching condition (3.11) for $\eta^{*}$ now shows that

$$
\lim _{z \rightarrow-h} \eta^{(1)}=\xi_{\alpha}^{(0)} \frac{\partial h}{\partial X_{\alpha}}+\frac{\nu^{1 / 2}}{\varepsilon} \frac{i \kappa_{\alpha} \xi_{\alpha}^{(0)}}{\gamma}
$$

Thus the outcome of this boundary layer expansion is to supply a bottom boundary condition for the interior variable $\eta^{(1)}$, which satisfies the interior equation (3.7a).

Next, the free surface boundary layer variables are defined by

$$
z^{*}=\frac{z}{\nu^{1 / 2}}, \quad \eta=\eta^{(i)}+\eta^{*}, \quad \xi_{\alpha}=\xi_{\alpha}^{(i)}+\nu^{1 / 2} \xi_{\alpha}^{*} \quad \text { and } \hat{p}=\hat{p}^{(i)}+\nu^{1 / 2} \hat{p}^{*}
$$

where the superscript index ( $i$ ) denotes the interior solution, defined by (3.1a, b). The use of the same notation for both boundary layers should cause no confusion, as the context will make it clear which boundary layer is being 
considered. The boundary layer equations are again $(3.9 \mathrm{a}, \mathrm{b}, \mathrm{c})$ with the omission of the terms proportional to $\partial h / \partial X_{\alpha}$. The boundary conditions are $(2.25 \mathrm{a}, \mathrm{b})$ which, in the boundary layer variables $(3.15)$, become $-\nu^{1 / 2} \hat{p}^{*}+g \nu \eta^{*}=a\left\{\varepsilon\left(\hat{p}^{(1)}-g \eta^{(1)}\right)+\nu 2 i \omega \kappa \operatorname{coth} \kappa h A\right\} \exp (i \theta) \quad$ on $z^{*}=0$, and

$$
\frac{\partial \xi_{\alpha}^{*}}{\partial z^{*}}=\left(-a 2 i \kappa_{\alpha} A\right) \exp (i \theta)+\text { c.c.. }
$$

The matching conditions are

$$
\lim _{z^{*} \rightarrow \infty}\left\{\xi_{\alpha}^{*}, \eta^{*}, p^{*}\right\}=0
$$

Equation (3.9c) with the matching conditions (3.17) shows that $\hat{p}^{*}$ is $O(v)$. Next, the solution to (3.9b) which is proportional to $\exp (i \theta)$, and satisfies the boundary condition (3.16b) and the matching conditions (3.17), is

$$
\xi_{\alpha}^{*}=-a 2 i \kappa_{\alpha} A \exp (i \theta) \frac{\exp \left(\gamma z^{*}\right)}{\gamma}+\text { c.c.. }
$$

Finally, $\eta^{*}$ is found from (3.9a) and (3.7) which give

$$
\eta^{*}=-\frac{a 2 i \kappa^{2} A}{\omega} \exp (i \theta) \exp \left(\gamma z^{*}\right)+\text { c.c.. }
$$

The boundary condition (3.16a) now shows that

$$
\lim _{z \rightarrow 0}\left(\hat{p}^{(1)}-g \eta^{(1)}\right)=-\frac{\nu}{\varepsilon} \frac{4 i g \kappa^{2} A}{\omega} .
$$

This is the free surface boundary condition for the interior variable $\eta^{(1)}$.

We have now established that the interior variable $\eta^{(1)}$ satisfies (3.7a) with the boundary conditions (3.14) and (3.20). A necessary and sufficient condition that this inhomogeneous boundary value problem have a solution is the compatibility condition

$$
\begin{array}{r}
\int_{-h}^{0}\left\{\eta^{(0)} \kappa^{2} F_{3}-\frac{\partial \eta^{(0)}}{\partial z}\left(\omega^{2} I+i \kappa_{\alpha} F_{\alpha}\right)\right\} d z+\left[\omega^{2} \frac{\partial \eta^{(0)}}{\partial z}\left(\eta^{(1)}-\frac{1}{g} \hat{p}^{(1)}\right)\right]_{z=0} \\
-\left[\omega^{2} \frac{\partial \eta^{(0)}}{\partial z} \eta^{(1)}\right]_{z=-h}=0 .
\end{array}
$$

This condition is derived by using the method of variation of parameters to solve (3.6a) and then applying the boundary conditions (see [7]). If we now substitute (3.3), (3.6), (3.14) and (3.20) into (3.21) we get

$$
\frac{\partial \mathscr{Q}}{\partial T}+\frac{\partial}{\partial X_{\alpha}}\left(V_{\alpha} \mathscr{Q}\right)+\sigma \mathscr{Q}=0,
$$


where

$$
\mathscr{Q}=\frac{2 g A^{2}}{\omega}
$$

and

$$
\sigma=\frac{\nu^{1 / 2}}{\varepsilon} \frac{2 \gamma \kappa}{\sinh 2 \kappa h}+\frac{\nu}{\varepsilon} 4 \kappa^{2},
$$

and $V_{\alpha}$ is the group velocity, $\partial \omega / \partial \kappa_{\alpha}$. In the absence of friction, $\sigma$ is zero, and (3.22a) is the equation for conservation of wave action (see [7], where the same equation is derived in more general circumstances); $\mathcal{Q}$ is the complex wave action. The coefficient $\sigma$ is the frictional decay factor [13]; if bottom friction dominates $\nu$ scales with $\varepsilon^{2}$ and only the first term in $(3.27 \mathrm{c})$ is required; in the deep water approximation $\nu$ scales with $\varepsilon$ and only the second term in (3.22c) is required. The universality of the conservation of wave action as the principle for determining the modulation of the wave amplitude is well known [2, 3]. In the context of water waves the Lagrangian method of deriving equation (3.22a) is relatively unusual although, as Andrews and McIntyre have pointed out, there is a very close connection between Lagrangian concepts and wave action.

\section{Evolution of the mean flow}

Now that the perturbation variables are known to $O(a)$ from (3.3), with the amplitude $A$ determined from (3.22a), the forcing terms in the mean flow equations may be evaluated to $O\left(a^{2}\right)$. The mean flow equations are either (2.15b) and (2.20b), with boundary conditions (2.23), (2.24) and (2.26) in the Lagrangian formulation, or (2.22) with boundary conditions (2.27) in the Eulerian formulation. Because the perturbation flow is irrotational in the interior it is more convenient to use the latter formulation. Indeed, when $\hat{u}_{i}$ is irrotational, the forcing term $R_{i}(2.22 \mathrm{c})$ becomes

$$
R_{i}=-\frac{\partial}{\partial x_{i}}\left\langle\frac{1}{2} \hat{u}_{j} \hat{u}_{j}\right\rangle,
$$

and the mean flow equation $(2.22 b)$ is simply

$$
\frac{\partial \bar{u}_{i}}{\partial t}+\frac{\partial}{\partial x_{i}}\left(\bar{p}+\left\langle\frac{1}{2} \hat{u}_{j} \hat{u}_{j}\right\rangle\right)=\nu \frac{\partial^{2} \bar{u}_{i}}{\partial x_{k} \partial x_{k}}
$$

Thus, in the interior, the effect of the waves on the Eulerian mean flow is simply an adjustment of the mean pressure field [1]. Since the mean flow depends only on $X_{\alpha}, T$ and $z$, the leading terms in the interior mean flow equations (2.22a) 
and (4.2) are

$$
\begin{aligned}
\frac{\partial \bar{u}_{\alpha}}{\partial X_{\alpha}}+\frac{\partial \bar{w}}{\partial z} & =0 \\
\frac{\partial \bar{u}_{\alpha}}{\partial T}+\frac{\partial \bar{q}}{\partial X_{\alpha}} & =\frac{\nu}{\varepsilon} \frac{\partial^{2} \bar{u}_{\alpha}}{\partial z^{2}}, \\
\frac{\partial \bar{q}}{\partial z} & =0
\end{aligned}
$$

and

$$
\bar{p}=\bar{q}+\frac{a^{2} \omega^{2} \cosh 2 \kappa(z+h)|A|^{2}}{\sinh ^{2} \kappa h} .
$$

Here we have replaced $\bar{w}$ by $\mathrm{e} \bar{w}$ as the continuity equation (4.3a) implies that $\bar{w}$ is $O(\varepsilon)$ relative to $\bar{u}_{\alpha}$. The continuity equation $(4.3 \mathrm{a})$ is also valid in both boundary layers; combined with the boundary condition that $\bar{w}=0$ at $z=-h$ (2.27a), it serves to specify $\bar{w}$ completely. Note that equation (4.3c) shows that $\bar{q}$ is independent of $z$ to leading order in $\varepsilon$. To find the mean flow, it is necessary to calculate the "Stokes corrections". For instance, from (2.21), it follows that

$$
u_{\alpha}^{s}=\frac{a^{2} 2 \omega \kappa_{\alpha} \cosh 2 \kappa(z+h)|A|^{2}}{\sinh ^{2} \kappa h},
$$

and

$$
\bar{w}^{s}=\frac{\partial}{\partial T}\left(\frac{a^{2} \kappa \sinh 2 \kappa(z+h)|A|^{2}}{\sinh ^{2} \kappa h}\right)-\frac{\partial}{\partial X_{\alpha}}\left(\frac{a^{2} \omega \kappa_{\alpha} \sinh 2 \kappa(z+h)|A|^{2}}{\kappa \sinh ^{2} \kappa h}\right) .
$$

In the bottom boundary layer, the forcing terms are no longer irrotational, and are given instead by (3.12a) and (3.13). Evaluating $R$, using (2.22c) we find that, to leading order,

$$
R_{\alpha}=\frac{\partial}{\partial z^{*}}\left\{-\frac{1}{\nu^{1 / 2}}\left\langle\hat{w} \hat{u}_{\alpha}\right\rangle-\frac{\varepsilon}{\nu^{1 / 2}} \frac{\partial h}{\partial X_{\alpha}}\left\langle\hat{u}_{\beta} \hat{u}_{\alpha}\right\rangle\right\}
$$

and

$$
R_{3}=O\left(a^{2} \nu^{1 / 2}\right)
$$

Recalling, from (3.8), that in the bottom boundary layer $\eta$, and here $\hat{w}$, is $O\left(\nu^{1 / 2}\right)$, we see that $R_{\alpha}$ is $O\left(a^{2}\right)$. Hence the mean flow equations in the bottom boundary layer are

$$
\frac{\partial^{2} \bar{u}_{\alpha}}{\partial z^{* 2}}+R_{\alpha}=0
$$


and

$$
\frac{\partial \bar{p}}{\partial z^{*}}=0
$$

These equations are to be solved subject to the boundary condition (2.27a) or

$$
\vec{u}_{\alpha}=0 \text { on } z^{*}=\mathbf{0} \text {. }
$$

Equation (4.6b) shows that $\bar{p}$ is constant throughout the boundary layer, and so is given by (4.3d) evaluated at $z=-h$. Then $\bar{u}_{\alpha}$ is found by integrating twice with respect to $z^{*}$, applying the boundary condition (4.7) and the matching condition $\partial \bar{u}_{\alpha} / \partial z^{*} \rightarrow 0$ as $z^{*} \rightarrow \infty$; this latter condition is required since $\partial \bar{u}_{\alpha} / \partial z$ is $O\left(a^{2}\right)$ in the interior, but equals $\nu^{-1 / 2} \partial \bar{u}_{\alpha} / \partial z^{*}$ in the boundary layer. The result is

$$
\begin{aligned}
\bar{u}_{\alpha}= & \frac{a^{2} \omega \kappa_{\alpha}|A|^{2}}{\sinh ^{2} \kappa h}\left\{-\gamma z^{*} \exp \left(-\gamma z^{*}\right)+[2+i \operatorname{sign} \omega]\left[1-\exp \left(-z^{*}\right)\right]\right. \\
& \left.-\frac{1}{2}\left[1-\exp \left(-(\gamma+\bar{\gamma}) z^{*}\right)\right]+\text { c.c. }\right\} .
\end{aligned}
$$

Next, letting $z^{*} \rightarrow \infty$ in (4.8) and matching with a limit as $z \rightarrow-h$ from the interior, we deduce that

$$
\lim _{z \rightarrow-h} \bar{u}_{\alpha}=\frac{a^{2} 3 \omega \kappa_{\alpha}|A|^{2}}{\sinh ^{2} \kappa h} .
$$

Equation (4.9) provides the bottom boundary condition for the interior mean flow equations (4.3), and was first derived by Longuet-Higgins [10]; an explanation of how the Reynolds stress $\left\langle\hat{w} \hat{u}_{\alpha}\right\rangle$ is responsible for the transfer of momentum across the boundary layer has been given by Longuet-Higgins in an appendix to the paper by Russell and Onsario [14]. The Stokes velocity $\bar{u}_{\alpha}^{S}$ can be computed from (2.21), (3.12a) and (3.13). Then the Lagrangian mean velocity is found from the sum of $\bar{u}_{a}$ and $\bar{u}_{\alpha}$ :

$$
\bar{u}_{\alpha}^{L}=\frac{a^{2} \omega \kappa_{\alpha}|A|^{2}}{\sinh ^{2} \kappa h}\left\{\frac{5}{2}-4 \exp \left(-\gamma z^{*}\right)+\frac{3}{2} \exp \left(-(\gamma+\bar{\gamma}) z^{*}\right)+\text { c.c. }\right\} \text {. }
$$

For the free surface boundary layer, the forcing terms are evaluated from (3.18) and (3.19). For this boundary layer it is more convenient to first calculate the Lagrangian mean flow. Following the method used for the perturbation variables, we put

$$
\bar{u}_{\alpha}^{L}=\bar{u}_{\alpha}^{L(i)}+\bar{u}_{\alpha}^{L^{*}} \text { and } \bar{p}^{L}=\bar{p}^{L(i)}+\bar{p}^{L^{*}} \text {, }
$$

where, as in (3.15), the superscript index (i) denotes the interior solution. Next, evaluating $R_{i}^{L}$ from (2.20c), (3.3), (3.18) and (3.19), we find that, to leading order,

$$
R_{\alpha}^{L}=R_{\alpha}^{L(i)}+a^{2} \nu^{1 / 2} \frac{\partial}{\partial z^{*}}\left\{\frac{8 \omega \kappa \kappa_{\alpha}|A|^{2}}{\tanh \kappa h}\left(\exp \left(\gamma z^{*}\right)+\text { c.c. }\right)\right\}
$$


and

$$
R_{3}^{L}=O\left(a^{2}\right)
$$

and so the boundary layer equations are

$$
\frac{\partial^{2} \bar{u}_{\alpha}^{L^{*}}}{\partial z^{* 2}}+R_{\alpha}^{L}-R_{\alpha}^{L(i)}=0
$$

and

$$
\frac{\partial \bar{p}^{L^{*}}}{\partial z^{*}}=0
$$

The matching conditions with the interior solution are that

$$
\lim _{z^{*} \rightarrow-\infty}\left\{\bar{u}_{\alpha}^{L^{*}}, \bar{p}^{L^{*}}\right\}=0 \text {. }
$$

The boundary conditions are (2.24) and $(2.26 \mathrm{a}, \mathrm{b})$. In boundary layer variables the latter two are

$$
-\bar{p}^{L(i)}-\bar{p}^{L^{*}}+g \bar{\zeta}^{L}=0 \quad \text { on } z^{*}=0
$$

and

$$
\frac{\partial \bar{u}_{\alpha}^{L(\iota)}}{\partial z}+\nu^{-1 / 2} \frac{\partial \bar{u}_{\alpha}^{L^{*}}}{\partial z^{*}}=0 \quad \text { on } z^{*}=0
$$

Now (4.13b) shows that $\bar{p}_{\alpha}^{L^{*}}$ is constant throughout the boundary layer and, from the matching conditions (4.14), we see that it is $O\left(a^{2} \nu^{1 / 2}\right)$. Equation (4.12a) shows that $\bar{u}_{a}^{L^{*}}$ is $O\left(a^{2} \nu^{1 / 2}\right)$; integrating (4.13b), and using the matching conditions (4.14), it follows that

$$
\frac{\partial \bar{u}_{\alpha}^{L^{*}}}{\partial z^{*}}=-a^{2} \nu^{1 / 2}\left\{\frac{8 \omega \kappa \kappa_{\alpha}|A|^{2}}{\tanh \kappa h}\left(\exp \left(\gamma z^{*}\right)+\text { c.c. }\right)\right\} \text {. }
$$

Application of the boundary condition (4.15b) now shows that

$$
\lim _{z \rightarrow 0} \frac{\partial \bar{u}_{\alpha}^{L}}{\partial z}=\frac{a^{2} 16 \omega \kappa \kappa_{\alpha}|A|^{2}}{\tanh \kappa h},
$$

where we have now omitted the superscript $(i)$ as the limit process refers to a limit from the interior. Equation (4.17) is one of the free surface boundary conditions for the interior mean flow equations and was first derived by Longuet-Higgins $[10,11]$. The present Lagrangian method of deriving (4.17) is similar to that employed by Ünlüata and Mei [16]. The remaining boundary conditions (2.24) and (4.15a) may be combined into

$$
\lim _{z \rightarrow 0}\left(\frac{\partial \bar{p}^{L}}{\partial T}-g \bar{w}^{L}\right)=0
$$

which is the second free surface boundary conditions for the interior mean flow equations. 
Since the interior mean flow equations (4.3) are cast in Eulerian variables, we must convert the boundary conditions (4.17) and (4.18) into their Eulerian counterparts. The interior Stokes velocity is given by (4.4a) and it is readily shown that (4.17) is replaced by

$$
\lim _{z \rightarrow 0} \frac{\partial \bar{u}_{\alpha}}{\partial z}=\frac{a^{2} 8 \omega \kappa \kappa_{\alpha}|A|^{2}}{\tanh \kappa h}
$$

Next, using (4.3d) and (4.4b) and evaluating $\bar{p}^{s}$ from (2.11c), it follows that (4.18) is replaced by

$$
\frac{\partial \bar{q}}{\partial T}-\lim _{z \rightarrow 0} g \bar{w}=\frac{\partial}{\partial T}\left\{\frac{a^{2} 2 g \kappa|A|^{2}}{\sinh 2 \kappa h}\right\}-\frac{\partial}{\partial X_{\alpha}}\left\{\frac{a^{2} 2 g^{2} \kappa_{\alpha}|A|^{2}}{\omega}\right\} .
$$

Also, it may be shown that the mean Eulerian displacement of the free surface is given by

$$
g \bar{\zeta}=\bar{q}-\frac{a^{2} 2 g \kappa|A|^{2}}{\sinh \kappa h} .
$$

Finally we note that the reason the free surface boundary layer is more readily analysed in Lagrangian terms is because the boundary layer correction $\bar{u}_{\alpha}^{L^{*}}$ is $O\left(\nu^{1 / 2}\right)$. This is not the case for the corresponding Eulerian boundary layer correction $\bar{u}_{\alpha}^{*}$, and consequently a treatment of the free surface boundary layer in Eulerian terms necessitates calculating $R_{\alpha}$ to two orders of magnitude.

\section{Solutions of the interior mean flow equations}

The interior mean flow equation is (4.3b) with the boundary conditions (4.9), (4.19) and (4.20). For the convenience of the reader we shall display these equations again

$$
\frac{\partial \bar{u}_{\alpha}}{\partial T}+\frac{\partial \bar{q}}{\partial X_{\alpha}}=\frac{\nu}{\varepsilon} \frac{\partial^{2} \bar{u}_{\alpha}}{\partial z^{2}} \quad \text { for }-h<z<0
$$

where

$$
\begin{gathered}
\bar{u}_{\alpha}=\frac{a^{2} 3 \omega \kappa_{\alpha}|A|^{2}}{\sinh ^{2} \kappa h} \quad \text { on } z=-h, \\
\frac{\partial \bar{u}_{\alpha}}{\partial z}=\frac{a^{2} 8 \omega \kappa \kappa_{\alpha}|A|^{2}}{\tanh \kappa h} \quad \text { on } z=0,
\end{gathered}
$$

and

$$
\frac{\partial}{\partial T}\left\{\frac{\bar{q}}{g}-\frac{a^{2} 2 \kappa|A|^{2}}{\sinh 2 \kappa h}\right\}+\frac{\partial}{\partial X_{\alpha}}\left\{\int_{-h}^{0} \bar{u}_{\alpha} d z+\frac{a^{2} 2 g \kappa_{\alpha}|A|^{2}}{\omega}\right\}=0
$$


This last equation is obtained from (4.20) after solving for $\bar{w}$ from (4.3a). In these equations $\omega$ and $\kappa_{\alpha}$ are known functions determined from (3.2) and (3.4), while the amplitude $A$ is determined from (5.1d). The dependent variables are $\bar{u}_{\alpha}$ and $\bar{q}$, which is independent of $z$. From (4.4a) it may be shown that the second term in brackets in (5.1d) is the mass transport

$$
M_{n}=\int_{-h}^{0} \bar{u}_{\alpha}^{L} d z=\int_{-h}^{0} \bar{u}_{\alpha} d z+\frac{a^{2} 2 g \kappa_{\alpha}|A|^{2}}{\omega} .
$$

Recalling that $\bar{\zeta}$ is given by (4.21), it follows that (5.1d) may be written in the form

$$
\frac{\partial \bar{\zeta}}{\partial T}+\frac{\partial M_{\alpha}}{\partial X_{\alpha}}=0
$$

Thus (5.1d) is simply the integrated equation for conservation of mass.

A steady solution of these equations is found by assuming that $\omega, \kappa_{\alpha}$ and $A$ are independent of $T$, and seeking a solution for which $\bar{u}_{\alpha}$ and $\bar{q}$ are also independent of $T$. We find that

$$
\bar{u}_{\alpha}=-\frac{\varepsilon}{2 \nu} \frac{\partial \bar{q}}{\partial X_{\alpha}}\left(h^{2}-z^{2}\right)+\frac{a^{2} 8 \omega \kappa \kappa_{\alpha}|A|^{2}}{\tanh \kappa h}(z+h)+\frac{a^{2} 3 \omega \kappa_{\alpha}|A|^{2}}{\sinh ^{2} \kappa h},
$$

and

$$
\frac{\partial}{\partial X_{\alpha}}\left\{-\frac{\varepsilon}{3 \nu} h^{3} \frac{\partial \bar{q}}{\partial X_{\alpha}}+\frac{a^{2} h^{2} 4 \omega \kappa \kappa_{\alpha}|A|^{2}}{\tanh \kappa h}+\frac{a^{2} h 3 \omega \kappa_{\alpha}|A|^{2}}{\sinh ^{2} \kappa h}+\frac{a^{2} 2 g \kappa_{\alpha}|A|^{2}}{\omega}\right\}=0 .
$$

The quantity in brackets in (5.4b) is the mass transport $M_{\alpha}$. If it is further supposed that $\omega, \kappa_{\alpha}$ and $A$ are also independent of $X_{\alpha}$ (which means neglecting the frictional decay factor $\sigma(3.22 \mathrm{c})$ ) and hence constants, and also equation (5.4b) is satisfied by equating $M_{\alpha}$ with zero, then (5.4a) is Longuet-Higgins' [10] conduction solution. For steady flows equating $M_{\alpha}$ with zero is appropriate when the waves are contained in a wave tank with a wave absorber at one end; however, in an infinite wave tank or in the ocean it may be more appropriate to satisfy (5.4b) by equating $M_{\alpha}$ with a non-zero constant (Ünlüata and Mei [16]).

Alternatively, suppose that $\omega$ and $\kappa_{\alpha}$ are constants and $A$ is independent of $X_{\alpha}$. Then, from the wave action equation (3.22a), $|A|^{2}=($ constant $) \exp (-\sigma T)$. Liu and Davis [9] obtained a solution of (5.1) under these hypotheses by further supposing that $\bar{u}_{\alpha}=$ (functions of $z$ ) $\exp (-\sigma T)$. However, their solution contains some anomalies because at certain depths no solution could be found. It is also difficult to see how a solution of this kind can be set up from a realistic initial state.

In general, the wave action equation (3.22a) shows that the amplitude $A$ will be a function of $X_{\alpha}$ and $T$, and we must accordingly seek solutions of (5.1) for 
which $\bar{u}_{\alpha}$ and $\bar{q}$ are likewise functions of $X_{\alpha}$ and $T$. In this case it will facilitate subsequent discussion if we put

$$
\bar{u}_{\alpha}=\frac{\partial \phi}{\partial X_{\alpha}}+v_{\alpha}+U_{\alpha}
$$

and

$$
\bar{q}=-\frac{\partial \phi}{\partial T}+Q
$$

where $\phi\left(X_{\alpha}, T\right)$ is independent of $z$ and satisfies the equation

$$
\frac{\partial^{2} \phi}{\partial T^{2}}-\frac{\partial}{\partial X_{\alpha}}\left(g h \frac{\partial \phi}{\partial X_{\alpha}}\right)=-\frac{\partial}{\partial T}\left(\frac{a^{2} 2 \kappa g|A|^{2}}{\sinh 2 \kappa h}\right)+\frac{\partial}{\partial X_{\alpha}}\left(\frac{a^{2} 2 g^{2} \kappa_{\alpha}|A|^{2}}{\omega}\right),
$$

while $v_{\alpha}$ satisfies the equations

$$
\frac{\partial v_{\alpha}}{\partial T}=\frac{\nu}{\varepsilon} \frac{\partial^{2} v_{\alpha}}{\partial z^{2}} \quad \text { for }-h<z<0,
$$

where

$$
v=\frac{a^{2} 3 \omega \kappa_{\alpha}|A|^{2}}{\sinh ^{2} \kappa h}-\frac{\partial \phi}{\partial X_{\alpha}} \quad \text { on } z=-h
$$

and

$$
\frac{\partial v_{\alpha}}{\partial z}=\frac{a^{2} 8 \omega \kappa \kappa_{\alpha}|A|^{2}}{\tanh \kappa h} \quad \text { on } z=0 .
$$

Equations (5.1) will then be satisfied if we choose $U_{\alpha}$ to satisfy the equations

$$
\frac{\partial U_{\alpha}}{\partial T}+\frac{\partial Q}{\partial X_{\alpha}}=\frac{\nu}{\varepsilon} \frac{\partial^{2} U_{\alpha}}{\partial z^{2}} \text { for }-h<z<0,
$$

where

$$
\begin{aligned}
U_{\alpha}=0 & \text { on } z=-h, \\
\frac{\partial U_{\alpha}}{\partial z}=0 & \text { on } z=0
\end{aligned}
$$

and

$$
\frac{\partial}{\partial T}\left(\frac{Q}{g}\right)+\frac{\partial}{\partial X_{\alpha}}\left\{\int_{-h}^{0} U_{\alpha} d z\right\}=-\frac{\partial}{\partial X_{\alpha}}\left\{\int_{-h}^{0} v_{\alpha} d z\right\}
$$

Here the potential $\phi$ represents the inviscid irrotational solution to (5.1) (that is, the solution which puts $\nu=0$, and ignores the boundary conditions $(5.1 \mathrm{~b}, \mathrm{c})$ ); since $\phi$ is independent of $z$, the term $\partial \phi / \partial X_{\alpha}$ in (5.5a) describes a depth-independent velocity field in the interior of the fluid. Hence $\phi$ is completely determined by equation (5.6), together with initial and boundary conditions. For the case where $\omega, \kappa_{\alpha}$ and $h$ are constants, equation (5.6) agrees with the result of 
Davey and Stewartson [5] who considered the equations governing the evolution of modulated water waves in the inviscid case. The term $v_{\alpha}$ represents the viscous correction to this inviscid solution which accounts for the boundary conditions $(5.1 \mathrm{~b}, \mathrm{c})$; equations (5.7) describe the diffusion of vorticity from the boundaries. We shall show below that on time scales $T$ of $O(1)$, or $t$ of $O\left(\varepsilon^{-1}\right), v_{\alpha}$ is confined to boundary layers of thickness $(\nu / \varepsilon)^{1 / 2}$. However, there is a non-zero mass transport associated with $v_{\alpha}$ and so the terms $U_{\alpha}$ and $Q$ are needed to satisfy the mass transport equation. The sequence for solving these equations is, first, to find $\phi$ from (5.6), after which $v_{\alpha}$ can be determined from (5.7), and finally $U_{\alpha}$ and $Q$ can be found from (5.8).

Before describing the solutions to (5.6), (5.7) and (5.8) we shall consider briefly the deep water approximation for which $\kappa h \rightarrow \infty$, the boundary conditions at $z=-h$ are ignored, and $\nu$ scales with $\varepsilon$. In this approximation the solution of (5.6) is

$$
\frac{\partial \phi}{\partial X_{\alpha}} \sim-\frac{a^{2} 2 \kappa_{\alpha} \omega|A|^{2}}{\kappa h}
$$

and so $\phi$ is $O\left((\kappa h)^{-1}\right)$. Then $v_{\alpha}$ is determined from (5.7a) and (5.7c) alone, while it may be shown from (5.8d) that $U_{\alpha}$ and $Q$ are $O\left((\kappa h)^{-1}\right)$. Thus $v_{\alpha}$ is the dominant term in $\bar{u}_{\alpha}$, and equations $(5.7 \mathrm{a}, \mathrm{c})$ show that no steady solution is achieved as vorticity diffuses further and further into the fluid (see Longuet-Higgins, [11]). The solution for $v_{\alpha}$ determined from $(5.7 \mathrm{a}, \mathrm{c})$ is given below in equation $(5.17 \mathrm{~b})$. Ultimately, of course, the diffusing vorticity will reach the lower boundary, and the deep water approximation fails.

When the deep water approximation is not made, $\nu$ scales with $\varepsilon^{2}$. In order to keep the analysis simple we shall consider only the case when $\omega, \kappa$ and the depth $h$ are constants, $\kappa_{\alpha}=0$, and the amplitude $A$ is given by

$$
|A|^{2}=F\left(T^{*}\right) \exp \left(-\frac{\sigma X}{V}\right) \text { with } T^{*}=T-\frac{X}{V},
$$

where $X=X_{1}, V$ is the $X_{1}$-component of group velocity, $T^{*}$ is a co-ordinate moving with the wave packet, $\sigma$ is the frictional decay factor $(3.21 \mathrm{c})$, and $F(T)$ is a specified function of $T$. Equation (5.10) describes the generation of a onedimensional wave packet at $X=0$, which (assuming $V$ is positive) propagates in the positive $X$-direction, and has a spatial rate of decay of $\sigma / V$. We shall further suppose that $F(T) \rightarrow 0$ as $T \rightarrow-\infty$ so that the wave packet is set up from a state of rest. In the sequel we shall illustrate our results with two special cases,

(i) $\quad F(T)=H(T)$

or

$$
\text { (ii) } \quad F(T)=\delta(T) \text {, }
$$


where $H(T)$ is the Heaviside function (that is, zero for $T \leqslant 0$ and one for $T>0$ ), while $\delta(T)$ is the Kroneker delta function. Case (i) represents the initiation of a uniform wavetrain at $T=0, X=0$, and is a simple model of a wave tank. Case (ii) is a crude but representative model of a wave packet of finite length.

We turn first to equation (5.6) for $\phi$. This is simply the inhomogeneous wave equation and may be solved by a number of classical methods. We shall employ a Laplace transform in time, using the initial conditions that $\phi \rightarrow 0$ and $\partial \phi / \partial T$ $\rightarrow 0$ as $T \rightarrow-\infty$, and the boundary conditions that $\phi \exp (\sigma X / V)$ is bounded for all $T$ as $X \rightarrow \pm \infty$. Then it may readily be shown that the solution of (5.6) is

$$
\phi=\frac{1}{2 \pi i} \int_{\Gamma} \mathcal{L}(\phi) \exp (s T) d s,
$$

where

$$
\mathcal{L}(\phi)=\frac{\mathcal{L}(F) \exp \left\{-\frac{(\sigma+s) X}{V}\right\}}{\left[\frac{g h}{V}(\sigma+s)^{2}-s^{2}\right]}\left\{\frac{a^{2} 2 g \kappa s}{\sinh 2 \kappa h}+\frac{a^{2} 2 g^{2} \kappa(\sigma+s)}{\omega V}\right\} .
$$

Here $\mathcal{L}(F)$ is the Laplace transform of $F(T)$, and $\Gamma$ is a contour parallel, and to the right of, the imaginary $s$-axis. In case (i), (5.11a), $\mathcal{L}(F)$ is $s^{-1}, \mathcal{L}(\phi)$ has a pole at the origin and, evaluating $\phi$ from (5.12a), it follows that

$$
\phi \sim \frac{a^{2} 2 g \kappa V}{\omega h \sigma} \exp \left(-\frac{\sigma X}{V}\right) \text { as } T^{*} \rightarrow \infty,
$$

or

$$
\frac{\partial \phi}{\partial X} \sim-\frac{a^{2} 2 g \kappa}{\omega h} \exp \left(-\frac{\sigma X}{V}\right) \text { as } T^{*} \rightarrow \infty .
$$

More generally, if $F(T) \rightarrow 1$ as $T \rightarrow \infty$, then $\mathcal{L}(F) \rightarrow s^{-1}$ as $s \rightarrow 0$, and (5.13a, b) follow. Equation (5.13b) is readily seen to be the steady solution of (5.6). Further, since $\partial \phi / \partial X$ is the inviscid part of the steady solution (5.5a), substitution into (5.2) shows that the mass transport of the inviscid part of the solution tends to zero as $T^{*} \rightarrow \infty$. Note that, although $\partial \phi / \partial X$ is the inviscid part of the solution, the result $(5.13 \mathrm{a}, \mathrm{b})$ is crucially dependent on the frictional decay factor $\sigma$ being non-zero. In case (i), if $\sigma$ is zero, it is readily shown from $(5.12 a, b)$ that

$$
\frac{\partial \phi}{\partial X}=\frac{1}{\left[1-\frac{g h}{V^{2}}\right]}\left\{\frac{a^{2} 2 g \kappa}{V \sinh 2 \kappa h}+\frac{a^{2} 2 g^{2} \kappa}{\omega V^{2}}\right\} H\left(T^{*}\right),
$$

which agrees with the inviscid result of Davey and Stewartson [5], and may be established directly from (5.6). Equation (5.14) multiplied by $\exp (-\sigma X / V)$ also describes the solution for case (i) when $\sigma$ is non-zero as $T^{*} \rightarrow 0+$. Thus, at the 
front of the wave packet $\left(T^{*} \rightarrow 0+\right), \partial \phi / \partial X$ is given by the inviscid solution (5.14) multiplied by $\exp (-\sigma X / V)$, but behind the front $\partial \phi / \partial X$ decays as $T^{*} \rightarrow \infty$ to the solution (5.13b). The instantaneous solution (5.14) is associated with a non-zero mass transport, but the ultimate solution $(5.13 \mathrm{~b})$ is associated with a zero mass transport. In case (ii), (5.11b), $\mathcal{L}(F)$ is 1 and, evaluating $\phi$ from $(5.12 a, b)$, it follows that

$$
\begin{aligned}
\phi= & \frac{\exp \left\{-\frac{\sigma X}{V}-\frac{\sigma \sqrt{g h} T^{*}}{(\sqrt{g h}-V)}\right\}}{2\left[\frac{\sqrt{g h}}{V}-1\right]}\left\{\frac{a^{2} 2 g \kappa}{\sinh 2 \kappa h}+\frac{a^{2} 2 g^{2} \kappa}{\omega \sqrt{g h}}\right\} H\left(T^{*}\right) \\
& +\frac{\exp \left\{-\frac{\sigma X}{V}-\frac{\sigma \sqrt{g h} T^{*}}{(\sqrt{g h}+V)}\right\}}{2\left[\frac{\sqrt{g h}}{V}+1\right]}\left\{\frac{-a^{2} 2 g \kappa}{\sinh 2 \kappa h}+\frac{a^{2} 2 g^{2} \kappa}{\omega \sqrt{g h}}\right\} H\left(T^{*}\right) .
\end{aligned}
$$

When $T^{*} \rightarrow \infty, \phi$ and $\partial \phi / \partial X \rightarrow 0$; as $T^{*} \rightarrow 0+, \partial \phi / \partial X$ is again given by (5.14) multiplied by $\exp (-\sigma X / V)$, with the Heaviside function replaced with the $\delta$-function. Thus the instantaneous solution at the front of the wave packet is analogous to case (i), but behind the front $\partial \phi / \partial X \rightarrow 0$. Finally we note that the complete solution for case (i) may be obtained from (5.15) by integrating with respect to $T^{*}$.

With $\phi$ determined, we may turn to equations (5.7) to find $v_{\alpha}$. With $|A|^{2}$ specified by (5.10), and the initial condition that $v_{\alpha} \rightarrow 0$ as $T \rightarrow-\infty$, the solution can be found using a Laplace transform in time. With $v_{2}=0$, and denoting $v_{1}$ by $v$, the Laplace transform of $v$ is

$$
\begin{aligned}
\mathcal{L}(v)= & {\left[\frac{a^{2} 8 \omega \kappa^{2} \mathcal{L}(F) \exp \left(-\frac{(\sigma+s) X}{V}\right)}{\tanh \kappa h}\right] \frac{\sinh \left\{\left(\frac{s E}{\nu}\right)^{1 / 2}(z+h)\right\}}{\left(\frac{s \varepsilon}{\nu}\right)^{1 / 2} \cosh \left\{\left(\frac{s \varepsilon}{\nu}\right)^{1 / 2} h\right\}} } \\
& +\left[\frac{a^{2} 3 \omega \kappa \mathcal{L}(F) \exp \left(-\frac{(\sigma+s) X}{V}\right)}{\sinh ^{2} \kappa h}-\mathcal{E}\left(\frac{\partial \phi}{\partial X}\right)\right] \frac{\cosh \left\{\left(\frac{s \varepsilon}{\nu}\right)^{1 / 2} z\right\}}{\cosh \left\{\left(\frac{s \varepsilon}{\nu}\right)^{1 / 2} h\right\}},
\end{aligned}
$$

and $v$ is then found from an inversion formula analogous to (5.12a). However, since $\nu$ scales with $\varepsilon^{2}, \sqrt{\nu} / \varepsilon$ is a small parameter of $O(\varepsilon)$, and on time scales $T^{*}$ 
of $O(1)$, the formula (5.16) may be simplified by replacing expressions such as $\cosh \left\{(s \varepsilon / \nu)^{1 / 2} h\right\}$ with $\frac{1}{2} \exp \left\{(s \varepsilon / \nu)^{1 / 2} h\right\}$ and so on. The Laplace inversion may then be performed explicitly, and the result is

$$
v \sim v_{f}+v_{h},
$$

where

$$
\begin{gathered}
v_{f}=-\int_{-\infty}^{T^{*}} \frac{\partial K}{\partial Z_{f}}\left(T^{*}-T^{\prime}, Z_{f}\right)\left(\frac{\nu}{\varepsilon}\right)^{1 / 2} \frac{a^{2} 8 \omega \kappa^{2} F\left(T^{*}\right)}{\tanh \kappa h} \exp \left(-\frac{\sigma X}{V}\right) d T^{\prime} \\
v_{h}=\int_{-\infty}^{T^{*}} K\left(T^{*}-T^{\prime}, Z_{h}\right) \frac{\partial}{\partial T^{\prime}}\left\{\frac{a^{2} 3 \omega \kappa F\left(T^{\prime}\right)}{\sinh ^{2} \kappa h} \exp \left(-\frac{\sigma X}{V}\right)-\frac{\partial \phi}{\partial X}\left(T^{\prime}, X\right)\right\} d T^{\prime} \\
K(T, Z)=\frac{2}{\sqrt{\pi}} \int_{Z / 2 \sqrt{T}}^{\infty} e^{-\lambda^{2}} d \lambda \\
Z_{f}=-\left(\frac{\varepsilon}{\nu}\right)^{1 / 2} z \text { and } Z_{h}=\left(\frac{\varepsilon}{\nu}\right)^{1 / 2}(z+h)
\end{gathered}
$$

Here $K(T, Z)$ is the complementary error function, and $Z_{f}$ and $Z_{h}$ are boundary layer co-ordinates at the free surface and bottom, respectively. The expression $v_{f}$ (5.17b) can be recognized as the solution of (5.7a) which satisfies only the boundary condition $(5.7 \mathrm{c})$ and describes the diffusion of vorticity from the free surface boundary layer; on a time scale $T^{*}$ of $O(1), v_{f}$ is only $O(\sqrt{(\nu / \varepsilon)})$ and the vorticity has penetration only a distance of $O(\sqrt{(\nu / \varepsilon)})$ into the interior. Note that $v_{f}$ is also the total solution for $v$ in the deep-water approximation. Similarly, the expression $v_{h}(5.17 \mathrm{c})$ is the solution of $(5.7 \mathrm{a})$ which satisfies only the bottom boundary condition (5.7b) and describes diffusion from the bottom boundary layer, the penetration distance again being $O(\sqrt{(\nu / \varepsilon)})$. For case (i), (5.11a), we may show that, for $T^{*}>0$,

$$
v_{f}=a^{2}\left(\frac{\nu}{\varepsilon}\right)^{1 / 2} \frac{8 \omega \kappa^{2} \exp \left(-\frac{\sigma X}{v}\right)}{\tanh \kappa h}\left\{2\left(\frac{T^{*}}{\pi}\right)^{1 / 2} \exp \left(-\frac{Z_{f}^{2}}{4 T^{*}}\right)-Z_{f} K\left(T^{*}, Z_{f}\right)\right\},
$$

and

$$
v_{h}=a^{2}\left\{\frac{3 \omega \kappa}{\sinh ^{2} \kappa h}+\frac{2 g k}{\omega h}\right\} \exp \left(-\frac{\sigma X}{V}\right) K\left(T^{*}, Z_{h}\right)\left[1+O\left(T^{*-1 / 2}\right)\right]
$$

In (5.18b) the error terms arise due to the fact that we have replaced $\partial \phi / \partial X$ by its asymptotic expression (5.13b) and error terms. Of course, these expressions 
are valid only for time scales $T^{*}$ of $O(1)$. In order to determine the limit as $T^{*} \rightarrow \infty$ we must return to (5.16). Clearly the limit $T^{*} \rightarrow \infty$ for $v$ takes place on time scales $T^{*}$ of $O\left(h^{2} / \nu\right)$, and is related to the time for the free surface vorticity to penetrate to the bottom boundary. Evaluating $\mathscr{L}(v)$ by $(5.16)$ as $s \rightarrow 0$, with $\mathcal{E}(F)$ equal to $s^{-1}$, and inverting the Laplace transform we find that

$$
v \rightarrow \frac{a^{2} 8 \omega \kappa^{2} \exp \left(-\frac{\sigma X}{V}\right)}{\tanh \kappa h}(z+h)+a^{2}\left\{\frac{3 \omega \kappa}{\sinh ^{2} \kappa h}+\frac{2 g \kappa}{\omega h}\right\} \exp \left(-\frac{\sigma X}{V}\right)
$$

as $T^{*} \rightarrow \infty$.

Note that the second term in (5.19) is also the limit as $T^{*} \rightarrow \infty$ of $v_{h}(5.18 \mathrm{~b})$. The reason for this is that the steady part of $v_{h}$ is independent of $z$ and so satisfies the free surface boundary condition as well; like $\partial \phi / \partial X, v_{h}$ becomes steady on time scales $T^{*}$ of $O(1)$. By contrast, $v_{f}(5.18 \mathrm{a})$ is not steady on these wave packet time scales, and requires the longer time scale of $O\left(\varepsilon h^{2} / \nu\right)$ to become steady. Huang [8] has drawn attention to the apparent paradox that the Longuet-Higgins conduction solution for the free surface velocity $(Z=0)$ increases linearly with depth when $\kappa h \gg 1$. This situation appears in (5.19a). However, the paradox is only apparent as (5.19a) holds only in the limit $T^{*} \rightarrow \infty$ with $h$ fixed; in other words the deep water approximation $\kappa h \gg 1$ for the mean flow holds only for times $T^{*}$ significantly less than $\varepsilon h^{2} / \nu$. A related apparent paradox occurs in (5.18a) which shows that the free surface velocity for times $T^{*}$ of $O(1)$ is proportional to $\sqrt{\left(\nu T^{*} / \varepsilon\right)}$ which increases indefinitely for large times. Again, the paradox is only apparent as (5.18a) holds only for times $T^{*}$ of $O(1)$, or, more precisely, $T^{*}$ significantly less than $\varepsilon h^{2} / \nu$. In shallow water, for which $\nu$ scales with $\varepsilon^{2}$, the contribution of $(5.18 a)$ to the free surface velocity is very small compared to (5.13b). For case (ii), (5.11b), we may show that, for $T^{*}>0$,

$$
v_{f}=a^{2}\left(\frac{\nu}{\varepsilon}\right)^{1 / 2} \frac{8 \omega \kappa^{2} \exp \left(-\frac{\sigma X}{V}\right)}{\tanh \kappa h} \frac{\exp \left(-\frac{Z_{f}^{2}}{4 T^{*}}\right)}{\sqrt{\pi} T^{*}},
$$

and

$$
v_{h}=a^{2}\left\{\frac{3 \omega \kappa}{\sinh ^{2} \kappa h}+\frac{2 g \kappa}{\omega h}\right\} \exp \left(-\frac{\sigma X}{V}\right) \frac{\partial K}{\partial T}\left(T^{*}, Z_{h}\right)\left[1+O\left(T^{*-1 / 2}\right)\right] .
$$

Both $v_{f}$ and $v_{h} \rightarrow 0$ as $T^{*} \rightarrow \infty$, and do so on time scales $T^{*}$ of $O(1)$. Due to the finite length of the wave packet there is insufficient time for the diffusion processes to penetrate further than a distance $O\left((\nu / \varepsilon)^{1 / 2}\right)$ into the interior.

Finally, we may turn to equations (5.8) for $U_{\alpha}$ and $Q$. We again employ Laplace transforms in time. With $U_{2}=0$, and denoting $U_{1}$ by $U$, the Laplace 
transforms of $U$ and $Q$ are given by

$$
\mathcal{L}(U)=-\frac{1}{s} \varrho\left(\frac{\partial Q}{\partial X}\right)\left[1-\frac{\cosh \left\{\left(\frac{s \varepsilon}{\nu}\right)^{1 / 2} z\right\}}{\cosh \left\{\left(\frac{s \varepsilon}{\nu}\right)^{1 / 2} h\right\}}\right]
$$

and

$$
\frac{s^{2}}{g} \mathcal{Q}(Q)-\left[h-\frac{\tanh \left\{\left(\frac{s \varepsilon}{\nu}\right)^{1 / 2} h\right\}}{\left(\frac{s \varepsilon}{\nu}\right)^{1 / 2}}\right] \frac{\partial^{2}}{\partial X^{2}} \mathcal{L}(Q)=-s \frac{\partial}{\partial X} \int_{-h}^{0} \mathcal{L}(v) d z .
$$

The right-hand side of $(5.21 \mathrm{~b})$ is known explicitly by substituting from (5.16). The equation (5.21b) can readily be solved explicitly, and $\mathcal{L}(Q)$ found; substitution into (5.21 a) gives $\mathcal{L}(U)$ explicitly. On time scales $T^{*}$ of $O(1)$, the formulae for the Laplace transforms simplify considerably and may be inverted. We find that the leading terms are

$$
U \sim-\int_{-\infty}^{T}\left\{1-K\left(T-T^{\prime}, Z_{h}\right)\right\} \frac{\partial Q}{\partial X}\left(T^{\prime}, X\right) d T^{\prime},
$$

where

$$
\begin{aligned}
h \frac{\partial Q}{\partial X} \sim & m(X, T) \\
& -\frac{\partial}{\partial T} \int_{0}^{\infty} \frac{1}{2}\{m(X+u \sqrt{g h}, T-u)-m(X+u \sqrt{g h}, T+u)\} d u,
\end{aligned}
$$

and

$$
\begin{aligned}
m(X, T)= & \left(\frac{\nu}{\varepsilon}\right)^{1 / 2} \frac{\partial}{\partial T} \int_{-\infty}^{T}\left[\pi\left(T-T^{\prime}\right)\right]^{-1 / 2}\left\{\frac{3 \omega \kappa|A|^{2}}{\sinh ^{2} \kappa h}-\frac{\partial \phi}{\partial X}\right\} d T^{\prime} \\
& +\frac{\nu}{h}\left\{\frac{8 \omega \kappa^{2}|A|^{2}}{\tanh \kappa h}+\frac{3 \omega \kappa|A|^{2}}{\tanh ^{2} \kappa h}-\frac{1}{h} \frac{\partial \phi}{\partial X}\right\}
\end{aligned}
$$

Here the dominant term in $U$ is the irrotational term for which $\partial U / \partial T=$ $-\partial Q / \partial X$, and the dominant term in $m$ is

$$
\frac{\partial}{\partial T} \int_{-h}^{0}\left(v_{f}+v_{h}\right) d z
$$

note that $m$ also contains a correction due to a bottom boundary layer contribution to $\int_{-h}^{0} U d z$. As $T^{*} \rightarrow \infty$, it may be shown that for, case (i), (5.11a), 
equations $(5.22 \mathrm{a}, \mathrm{b}, \mathrm{c})$ reduce simply to

$$
\begin{aligned}
\frac{h \partial Q}{\partial X} & \sim a^{2}\left(\frac{\nu}{\varepsilon}\right)^{1 / 2} \cdot \frac{1}{\sqrt{\pi} T^{*}}\left\{\frac{3 \omega \kappa}{\sinh ^{2} \kappa h}+\frac{2 g k}{\omega h}\right\} \exp \left(-\frac{\sigma X}{V}\right) \\
& +a^{2} \frac{\nu}{\varepsilon}\left\{\frac{8 \omega \kappa^{2}}{\tanh \kappa h}+\frac{3 \omega \kappa}{\tanh ^{2} \kappa h}+\frac{2 g}{\omega h^{2}}\right\} \exp \left(-\frac{\sigma X}{V}\right) \text { as } T^{*} \rightarrow \infty,
\end{aligned}
$$

and

$$
\begin{aligned}
U \sim & -\frac{a^{2}}{h}\left(\frac{\nu}{\varepsilon}\right)^{1 / 2}\left\{\frac{3 \omega \kappa}{\sinh ^{2} \kappa h}+\frac{2 g \kappa}{\omega h}\right\} \exp \left(-\frac{\sigma X}{V}\right) \\
& \times\left[2\left(\frac{T^{*}}{\pi}\right)^{1 / 2}\left\{1-\exp \left(-\frac{Z_{h}^{2}}{T^{*}}\right)\right\}+Z_{h} K\left(T^{*}, Z_{h}\right)\right] \\
& -\frac{a^{2}}{h} \frac{\nu}{\varepsilon}\left\{\frac{8 \omega \kappa^{2}}{\tanh \kappa h}+\frac{3 \omega \kappa}{h \sinh ^{2} \kappa h}+\frac{2 g \kappa}{\omega h^{2}}\right\} \exp \left(-\frac{\sigma X}{V}\right)\left[T^{*}\left\{1-K\left(T^{*}, Z_{h}\right)\right\}\right. \\
& \left.+Z_{h}\left(\frac{T^{*}}{\pi}\right)^{1 / 2} \exp \left(-\frac{Z_{h}^{2}}{4 T^{*}}\right)-\frac{1}{2} Z_{h}^{2} K\left(T^{*}, Z_{h}\right)\right]
\end{aligned}
$$

Equation (5.24a) for $h \partial Q / \partial X$ is simply $m(5.22 \mathrm{~b})$ as $T^{*} \rightarrow \infty$; then from (5.22a) and (5.24a) it follows that equation (5.24b) for $U$ is associated with zero mass transport for the sum $v+U$. These formulas are valid only for wave packet time scales when $T^{*}$ is $O(1)$. On the longer diffusive time scales, when $T^{*}$ is $O\left(\varepsilon h^{2} / \nu\right)$, the limit as $T^{*} \rightarrow \infty$ must be found from the exact expression $(5.21 \mathrm{a}, \mathrm{b})$. For case (i), (5.11a), we find that

$$
U \rightarrow-\frac{\varepsilon}{2 \nu} \frac{\partial Q}{\partial X}\left(h^{2}-z^{2}\right) \text { as } T^{*} \rightarrow \infty
$$

and

$$
\frac{\varepsilon}{3 \nu} h^{3} \frac{\partial Q}{\partial Z} \rightarrow a^{2}\left\{\frac{h^{2} 4 \omega \kappa^{2}}{\tanh \kappa h}+\frac{h 3 \omega \kappa}{\sinh ^{2} \kappa h}+\frac{2 g \kappa}{\omega}\right\} \exp \left(-\frac{\sigma X}{V}\right) \text { as } T^{*} \rightarrow \infty .
$$

Thus, on the diffusive time scale, the total solution $T^{*} \rightarrow \infty$ is the sum of (5.13b), (5.19) and (5.25b), which may be recognized as the Longuet-Higgins conduction solution, with zero mass transport. For case (ii), (5.11b), it is sufficient to calculate $Q$ and $U$ for $T^{*}$ of $O(1)$, and the solutions may be obtained from $(5.22 \mathrm{a}, \mathrm{b})$ by differentiating with respect to $T$. For this case of a wave packet of finite length, $U$ and $Q$ remain $O(\sqrt{(\nu / \varepsilon)})$ uniformly in $T^{*}$ as $T^{*} \rightarrow \infty$.

Russell and Onsorio [14] conducted a series of experiments in a wave tank on the generation of mean flows by a uniform wave train. Their observations were made over a period of from one to seven hours after the initiation of the wave 
train. For a time of one hour the penetration distance of a boundary layer, $\sqrt{(\nu \varepsilon)}$, is $6 \mathrm{~cm}$; after four hours it is $12 \mathrm{~cm}$. Since the depth of their wave tank was $50.8 \mathrm{~cm}$, this suggests that their observations were made on the wave packet time scale when $T^{*}$ is $O(1)$. In this case the approximate solution to describe their experimental results for the mass transport velocity is

$$
\bar{u}^{L} \sim \bar{u}^{s}+\frac{\partial \phi}{\partial X}+v+U
$$

where $\bar{u}^{s}$ is the Stokes velocity (4.4a), $\partial \phi / \partial X$ is given by $(5.13 \mathrm{~b}), v$ is given by $(5.18 \mathrm{a}, \mathrm{b})$, and $U$ is given by (5.24b). Here $\partial \phi / \partial X, v$ and $U$ have been evaluated on wave packet time scales, but using the limit as $T^{*} \rightarrow \infty$; this can be regarded as intermediate between the wave packet time scale and the diffusive time scale for which $T^{*}$ is $O\left(\varepsilon h^{2} / \nu\right)$. It can be shown that using the asymptotic expressions (5.13b), (5.18a, b) and (5.25b) rather than the exact expressions (5.12b), $(5.17 \mathrm{~b}, \mathrm{c})$ and $(5.22 \mathrm{~b})$ is justified for times $t$ greater than $\frac{1}{4}$ hour for the two cases described in Figs. 1 and 2 below. A more direct method of checking whether (5.27) is an adequate description of the experimental results is to compute

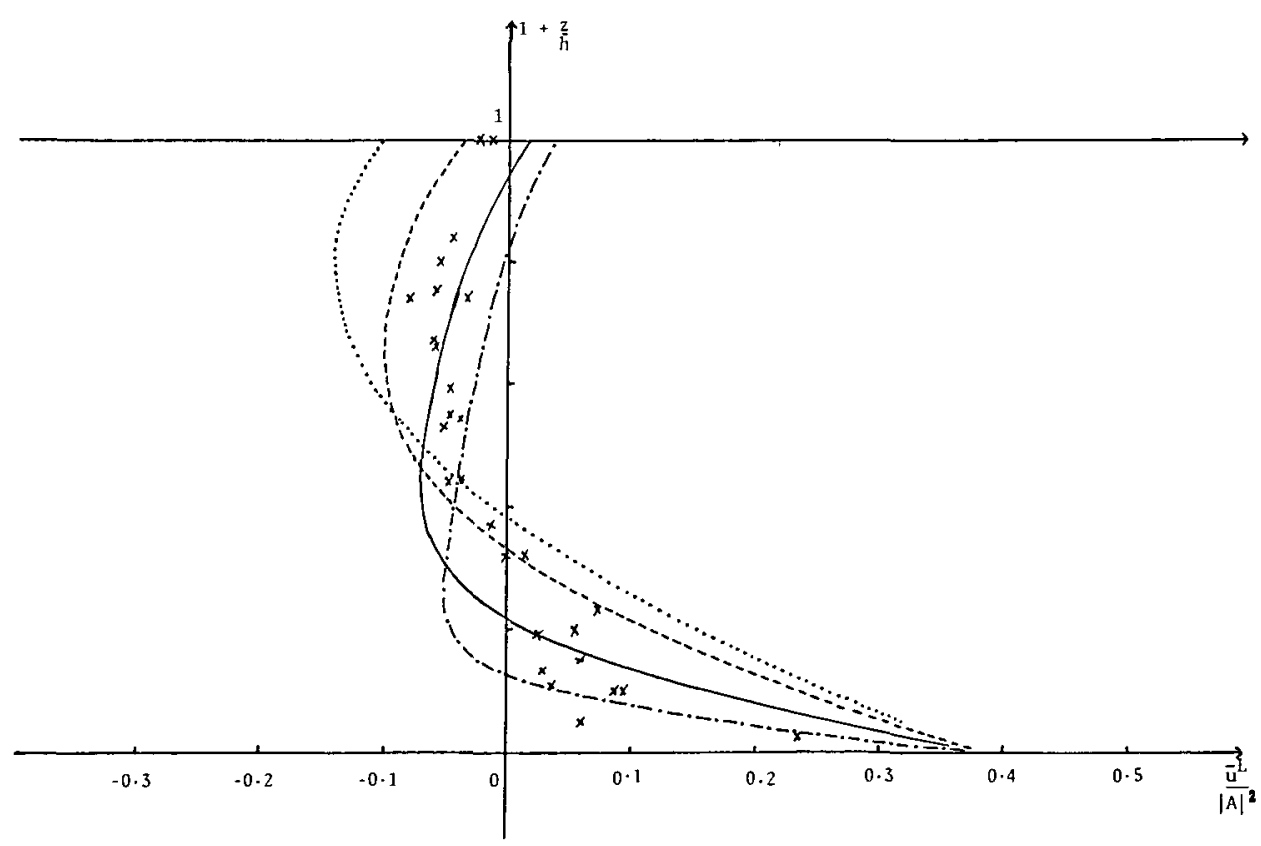

Figure 1. Graphs of the Lagrangian mean flow (5.26) for $\kappa h=0.5$ in a channel of depth $h=50.8 \mathrm{~cm}$.

- - . the present analysis at $t=\frac{1}{4}$ hour $(V(v t)=3 \mathrm{~cm}$.), the present analysis at $t=1$ hour $(V(\nu t)=6 \mathrm{~cm}$.), the present analysis at $t=4$ hours $(V(\nu t)=12 \mathrm{~cm}$.),

$X$, data of Russell and Onsorio [14] for an amplitude of $11.7 \mathrm{~cm}$. 


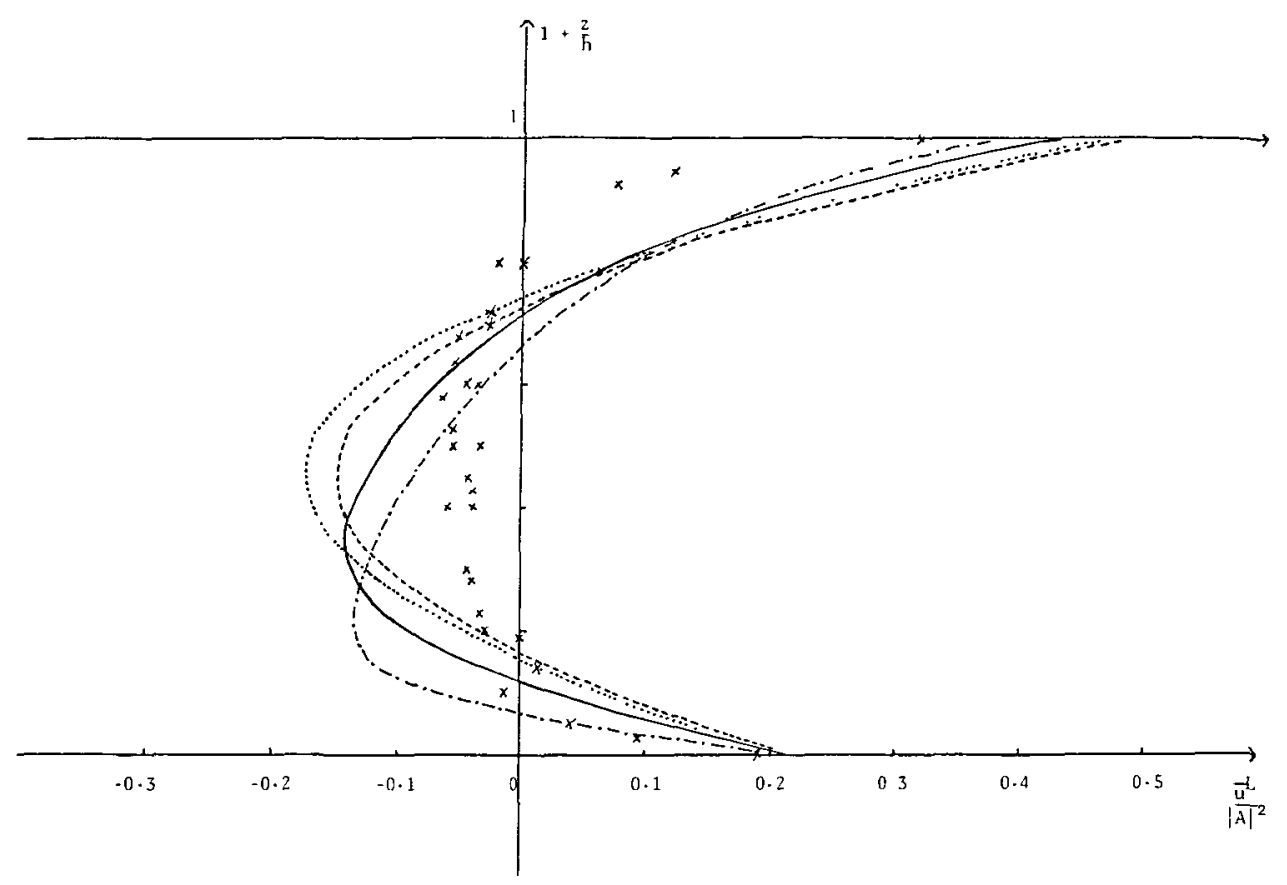

Figure 2. As for Fig. 1, with $\kappa h=1.25$.

$\partial v_{h} / \partial z$ and $\partial U / \partial z$ at $z=0$, and $v_{f}$ at $z=-h$, as (5.27) would be exact if all these terms were zero; we find that they have relative errors of $O\left(10^{-7}\right)$ for a time $t=1$ hour; however, at $t=4$ hours, $v_{f}$ at $z=-h$ has a relative error of $15 \%$, while $\partial v_{h} / \partial z$ and $\partial U / \partial z$ at $z=0$ have relative errors of $O\left(10^{-3}\right)$. Also since $\left(\bar{u}^{S}+\partial \phi / \partial X\right)$ and $(v+U)$ both separately produce zero mass transport, the solution (5.26) has zero mass transport, and is thus consistent with the experimental situation in a wave tank. The solutions considered in this section are for an infinite range of $X,-\infty<X<\infty$; a more exact analysis for $0<X<L$ and condition of zero mass transport at $X=0$ and $X=L$ has been performed by us, and we can show that, as $L \rightarrow \infty$, the results agree with those described by (5.26). Figs. 1 and 2 show graphs of the solution (5.26) for the two cases $h=0.5$ and 1.25 respectively, at the various times $t=\frac{1}{4}$ hour, 1 hour and 4 hours; also shown are the Longuet-Higgins conduction solution and the experimental points of Russell and Onsorio [14]. The graphs show that the experimental points generally lie closer to the graph (5.26) at times $t=1$ hour or $t=4$ hours, than to the graph of the Longuet-Higgins conduction solution. Russell and Onsorio [14] commented that the mean flow was observed to be steady after about one hour, whereas the theoretical curves in Figs. 1 and 2 are still evolving to the Longuet-Higgins conduction solution. However, the theoretical curves at 
' $=1$ hour and $t=4$ hours are quite close together, and the discrepancy may be due to experimental scatter. In the case of Fig. 2, where the experimental points show significant departure from all the theoretival curves, the discrepancy may be due to the neglect of the nonlinear advective terms in the mean flow equations (the mean flow Reynolds numbrr, although small in both cases, was larger in the case of Fig. 2 than in Fig. 1).

\section{References}

[1] D. G. Andrews and M. E. McIntyre, "An exact theory of nonlinear waves on a Lagrangianmean flow", J. Fluid Mech. 89 (1978), 609-646.

[2] D. G. Andrews and M. E. McIntyre, "On wave-action and its relatives", J. Fluid Mech. 89 (1978), 647-664.

[3] F. P. Bretherton and C. J. R. Garrett, "Wave trains in inhomogeneous moving media", Proc. Roy. Soc. 302A (1969), 529-554.

[4] M.-S. Chang, "Mass transport in deep-water leng-crested random gravity waves", $J$. Geophys. Res. 74 (1969), 1515-1536.

[5] A. Davey and K. Stewartson, "On three-dimensional packets of surface waves", Proc. Roy. Soc. 338A (1974), 101-110.

[6] B. D. Dore, "On mass transport velocity due to progressive waves", Quart. J. Mech. Appl. Maths. 30 (1977), 157-173.

[7] R. Grimshaw, "Mean flows induced by internal gravity wave packets propagating in a shear flow", Phil. Trans. Roy. Soc. 292A (1979), 391-417.

[8] N. E. Huang, “Mass transport induced by wave motion", J. Mar. Res. 34 (1970), 35-50.

[9] A.-K. Liu and S. H. Davis, "Viscous attenuation of mean drift in water waves", J. Fluid Mech. 81 (1977), 63-84.

[10] M. S. Longuet-Higgins, "Mass transport in water waves", Phil. Trans. Roy. Soc. 245A (1953), 535-581.

[11] M. S. Longuet-Higgins, "Mass transport in the boundary layer at a free oscillating surface", $J$. Fluid Mech. 8 (1960), 293-306.

[12] O. S. Madsen, "Mass transport in deep-water waves", J. Phys. Ocean. 8 (1978), 1009-1015.

[13] O. M. Phillips, The dynamics of the upper ocean (Cambridge University Press, 1969), Section 3.4.

[14] R. C. H. Russell and J. D. C. Onsorio, "An experimental investigation of drift profiles in a closed channel", Proc. 6th Conf. Coastal Eng., Miami (1957), 171-193.

[15] G. G. Stokes, "On the theory of oscillating waves", Trans. Camb. Phil. Soc. 8 (1847), 441-455.

[16] U. Unlüata and C. C. Mei, "Mass transport in water waves", J. Geophys. Res. 75 (1970), 7611-7618.

Department of Mathematics

University of Melbourne

Parkville

Victoria 3052 